\title{
Effect of heterogeneity on the elastic properties of auxetic materials
}

\author{
N. Gaspar, ${ }^{\text {a) }}$ C. W. Smith, and K. E. Evans \\ School of Engineering, University of Exeter, Exeter, Devon EX4 4QF, United Kingdom
}

(Received 27 May 2003; accepted 6 August 2003)

\begin{abstract}
Auxetic materials are gaining practical interest for their unusual and sometimes extreme mechanical response. The process of modeling these materials so far has highlighted a number of microstructural properties that are key to these materials. However these models often rely on the assumption of homogeneity and order within the materials. Practically, a homogeneous auxetic material such as foam is unlikely to be manufactured. This work seeks to analyze the effect of fluctuations within the microstructure of the material. Numerical results show the effect of fluctuations in an auxetic granular substance and analytical work indicates the relation between microscale fluctuations and the elastic moduli for a general auxetic material. (C) 2003 American
\end{abstract} Institute of Physics. [DOI: 10.1063/1.1614847]

\section{INTRODUCTION}

Auxetic materials, that is materials with a negative Poisson's ratio, have long been recognized as a theoretical possibility. ${ }^{1}$ A recent review of such materials is found in Evans and Alderson. ${ }^{2}$ Using isotropic elasticity, the constraint of stability on strain energy gives the following limits to the Poisson's ratio $\nu$

In two dimension

$-1<\nu<1$,

in three dimension

$$
-1<\nu<\frac{1}{2}
$$

The ratio between the bulk modulus $\kappa$, and shear modulus $\mu$, can also be expressed in terms of the Poisson's ratio

In two dimension

$$
\frac{\mu}{\kappa}=\frac{1-\nu}{1+\nu},
$$

in three dimension

$$
\frac{\mu}{\kappa}=\frac{3(1-2 \nu)}{1+\nu} .
$$

The limits in Eq. (1) have a physical interpretation based on Eq. (3). The negative limit indicates a material that cannot change shape, the positive limit indicating a material that cannot change volume. Where anisotropy can occur the Poisson's ratio has to be qualified to identify the axis and is then not bound by these limits. ${ }^{3-5}$ For example, granular media display $\nu>1$ under deviatoric loading; a phenomenon known as dilation that has only recently be adequately modeled. ${ }^{6}$ Conversely, some auxetic materials can produce a Poisson's ratio as large as -12 . $^{7}$

In 1987, Lakes $^{8}$ fabricated a polymeric foam that exhibited a negative auxetic behavior. There are many other materials (real and hypothetical) that can also exhibit auxetic

\footnotetext{
a) Author to whom correspondence should be addressed; electronic mail: n.gaspar@physics.org
}

behavior. An (incomplete) list follows: polymeric foams; ${ }^{8}$ expanded polytetrafluoroethylene, ${ }^{7}$ cubic elemental solids, rare gases, many hypothetical carbon structures, $\alpha$-crystobalite, zeolites and other crystal systems; ${ }^{4}$ keyed brick structures $;{ }^{2}$ ultrahigh molecular weight polyethylene ${ }^{9}$ polypropolyene (PP) ${ }^{10}$ auxetic granular materials; ${ }^{11}$ crumpled paper/entropic materials: membranes; ${ }^{12}$ skin; ${ }^{13}$ and tethered networks. ${ }^{12}$

Qualitatively, the constitutive properties and microstructure vary greatly between auxetic materials. Some, such as the $\alpha$-crystobalite or zeolites have a tightly constrained regular geometric arrangement that is required for the auxetic property. Materials such as polymeric foams or polypropylene have an array of constitutive elements whose properties vary randomly through the material. Length scales too can vary from the nanometer for molecular auxetics to the order of a meter for keyed-brick structures. ${ }^{2}$ This variability in auxetic materials makes theoretical modeling a complex task. Practically it leads to specific models for each type of material. It is unlikely and unnecessary that a single microstructural model can cover the full range of materials however it is reasonable to investigate some general properties that will reflect on a broad range of auxetic materials.

Classical linear elasticity predicts that auxetic materials have advantages over conventional materials. Hertz indentation theory predicts the radius of indentation to be proportional to $\left(1-\nu^{2}\right)^{1 / 3}$ for a hard ball indenter. ${ }^{14} \mathrm{~A}$ conventional foam has a Poisson's ratio of about 0.3 but auxetic foams have achieved $\nu=-0.76$. Hence the auxetic material approaches the limit of zero radius of indentation. The large ratio of shear modulus to bulk modulus in Eq. (4) is another mechanical property that has already been utilized in the design of MAGNOX reactors. In this case the material has a large resistance to earthquake shocks (shear loading) but a low resistance to thermal expansion (bulk loading). With these advantages in mind there are many possible uses for materials with negative Poisson's ratio. A problem that has so far resisted large-scale manufacturing is the current inability to make quantities of auxetic materials with consistent properties at viable costs in a high volume manufacturing 
scenario. If this can be overcome then potential applications range from snap fasteners to orthopedic cushioning and ballistics. ${ }^{15}$

Materials in the real world inevitably have imperfections or random variations in material properties. Part of the problem of manufacture is knowing how much these variations must be avoided or reduced to manufacture materials with predictable large scale properties. The majority of the modeling work to date utilizes a unit cell for the microscale for the model and assumes that the bulk material is a homogeneous tesellating collection of these cells. This article addresses the difference between a homogeneous model and a fluctuating or heterogeneous material by employing a homogenization technique that has been successfully used in granular mechanics. ${ }^{3}$ Numerical results are obtained that show the difference between a homogeneous material and a heterogeneous one. Then an analytic expression is produced that relates the correction for heterogeneity to the level of fluctuations within the material.

\section{FOAM AND GRANULAR MODELS}

Conventional models for a two dimensional (2D) foam center around a hexagonal cell shape. For topological stability $^{16}$ a $2 \mathrm{D}$ network should contain on average three contacts per node or six sides per cell, hence a hexagonal cell structure is well founded. To convert a physical foam sample from conventional to auxetic behavior it is compressed and heated in a prescribed manner. This process makes significant changes to the microstructure that bring about the change in elastic properties. ${ }^{14}$ Existing models of auxetic foam reflect this process by considering an altered cell model. Masters and Evans ${ }^{17}$ use a re-entrant cell or hourglass shape while Smith et al. ${ }^{18}$ consider a regular network that has certain connections broken.

In both cases elastic properties are ascribed to the unit cell. This is calculated from assumed elastic properties of the ribs that make up the cells, along with the geometry of the cell and the symmetries that makes the cell tessalate. In both models mentioned above there are parameters that help adjust the model to match experimental results. Masters and Evans ${ }^{17}$ identify a re-entrant cell structure that is nearly isotropic while Smith et al. ${ }^{18}$ are able to model the strain dependent behavior. Both models demonstrate some important microstructural considerations in the mechanics of conventional and auxetic foams but leave an important question unanswered. Is the microstructure of the foam successfully being modeled to reproduce the macroscopic effects or are the models simply presenting a possible homogeneous microstructure that has the same properties as a large sample of real foam? The difference matters when the model is asked to predict behavior beyond known data.

Another model of an auxetic material is that of a granular medium. ${ }^{19,11}$ For this medium to be auxetic the tangential interaction between grains has to be larger than the normal interaction. If $k_{N}$ and $k_{T}$ are the normal and tangential elastic interactions between grains and $\xi=k_{T} / k_{N}$ is the interaction ratio, then the Poisson's ratio is given by Eqs. (5) and (6).
From these equations auxetic behavior is seen to occur for $\xi>1$

3D material:

$$
\nu=\frac{1-\xi}{4+\xi},
$$

2D material:

$$
\nu=\frac{1-\xi}{3+\xi} .
$$

While this model of a strict granular material is hypothetical since naturally occurring granular materials do not possess this interaction, the theoretical concept can be applied to other materials. For instance, molecular structures can have a larger resistance to tangential than normal displacements. Beyond that application of "unusual grains" is a more general application. What actually is modeled is a random array of points interacting through noncentral forces. Other materials that can be described in this way are foams. Rothenburg et al. give a good account of how their random network model describes auxetic behavior in foams. They suggest that the buckled beams that are observed in auxetic foams have a weakened normal stiffness due to the buckle. Taking this further, results will be drawn from granular mechanics to shed light on auxetic materials in particular auxetic foams.

\section{MEAN-FIELD APPROXIMATIONS}

In a continuous material with stress $\sigma$, and strain $\epsilon$, connected by an elastic stiffness $\mathbf{Z}$ or compliance $\mathbf{C}=\mathbf{Z}^{-1}$ (the inverse of a fourth ordered tensor is defined as $(\mathbf{I})_{a b c d}^{-1} \mathbf{I}_{c d e f}$ $=\boldsymbol{\delta}_{a e} \boldsymbol{\delta}_{b f}$ ) and with position variable $\mathbf{x}$, the static equilibrium can be expressed as a zero divergence of stress [Eqs. (7)(9)]. This is arrived at by considering the force balance on a small unit volume

$$
\frac{\partial \boldsymbol{\sigma}_{a b}}{\partial x_{b}}=0,
$$

or

$$
\frac{\partial Z_{a b c d} \boldsymbol{\epsilon}_{c d}}{\partial x_{b}}=0,
$$

or

$$
\frac{\partial(\mathbf{C})_{a b c d}^{-1} \boldsymbol{\epsilon}_{c d}}{\partial x_{b}}=0 .
$$

In the case of a homogeneous material the variables $\boldsymbol{\epsilon}$ and $\mathbf{Z}$ are a constant function of position $\mathbf{x}$, and Eqs. (7)-(9) are trivially satisfied. For a heterogeneous material in equilibrium the strain and elastic moduli become functions of position $\mathbf{x}: \boldsymbol{\epsilon}(\mathbf{x})$ and $\mathbf{Z}(\mathbf{x})$. The local constitutive properties are

$$
\boldsymbol{\sigma}_{a b}(\mathbf{x})=Z_{a b c d}(\mathbf{x}) \boldsymbol{\epsilon}_{c d}(\mathbf{x}) .
$$

The aim is then to find $\mathbf{Z}^{\text {eff }}$ such that Eq. (11) is satisfied. The over-bar indicates a volume average

$$
\sigma_{a b}=Z_{a b c d}^{\mathrm{eff}} \bar{\epsilon}_{c d} .
$$


How $\mathbf{Z}^{\text {eff }}$ is arrived at from the field of $\mathbf{Z}(\mathbf{x})$ is the process of homogenization. The simplest methods are to calculate the arithmetic average

$$
\mathbf{Z}^{\text {eff } 1}=\frac{1}{V} \int \mathbf{Z}(\mathbf{x})
$$

or the harmonic average

$$
\left(\mathbf{Z}^{\mathrm{eff} 2}\right)^{-1}=\frac{1}{V} \int(\mathbf{Z}(\mathbf{x}))^{-1} .
$$

These two provide upper and lower bounds, effectively known as the mean strain and mean stress estimates since they are arrived at by assuming the strain (or stress) throughout the material is equal to the mean of that quantity. Alternative titles are the Voigt and Reuss estimates, respectively. A consequence of these mean-field approximations is that Eq. (10) is not satisfied. In the case of a mean-strain approximation equilibrium at a local scale is rejected, while in the case of a mean-stress approximation, the strain field is not uniquely defined.

Fortes and Ashby ${ }^{20}$ find Reuss and Voigt limits for a 2D cellular material. Using a honeycomb model with cell wall bending only the local Young's modulus $E_{U}$ is given by

$$
E_{U}=\frac{4 E_{S}}{\sqrt{(3)}}\left(\frac{t_{0}}{l_{0}}\right)^{3}
$$

where $t_{0}$ and $l_{0}$ are thickness and length respectively of cell walls and $E_{S}$ is the Young's modulus of the material that makes up the cell walls. Mean stress and mean strain techniques are applied to obtain the following limits.

$$
E_{\text {Reuss }} \leqslant E_{U} \leqslant E_{\text {Voigt }},
$$

mean stress estimate:

$$
\frac{E_{\text {Reuss }}}{E_{U}}=1-\frac{5}{2} \delta^{2}(l)-2 \delta^{2}(t),
$$

mean strain estimate:

$$
\frac{E_{\text {Voigt }}}{E_{U}}=1+\delta^{2}(l)+\delta^{2}(t),
$$

where $\delta(x)$ is the half width of the distribution of a statistical variable $x$ normalized on the average $\bar{x}$. It is difficult to utilize these results as only the Young's modulus is calculated and then only for a conventional hexagonal cell that in general does not exhibit auxetic behavior that this work is interested in. What is significant that Fortes and Ashby highlight is that the spread in the Young's modulus varies with square of the local fluctuations as measured by $\delta(\cdot)$.

\section{HOMOGENIZATION}

Position dependent variables can be separated into volume averaged and fluctuation quantities. The latter are signaled by a superscript ' + ' thus: $A(\mathbf{x})=\bar{A}+A^{+}$. Strictly speaking the fluctuation quantity should be written as a function of position $\mathbf{x}, A^{+}(\mathbf{x})$. For convenience this will be assumed. Applying the separation to Eq. (10) gives

$$
\begin{aligned}
\bar{\sigma}_{a b} & =\overline{Z_{a b c d}(\mathbf{x}) \boldsymbol{\epsilon}_{c d}(\mathbf{x})} \\
& =\overline{\bar{Z}_{a b c d} \overline{\boldsymbol{\epsilon}}_{c d}}+\overline{Z_{a b c d}^{+} \boldsymbol{\epsilon}_{c d}^{+}}+\overline{\bar{Z}_{a b c d} \overline{\boldsymbol{\epsilon}}^{+}}+\overline{Z_{a b c d}^{+}+\overline{\boldsymbol{\epsilon}}_{c d}} \\
& =\bar{Z}_{a b c d} \overline{\boldsymbol{\epsilon}}_{c d}+\overline{Z_{a b c d}^{+} \bar{\epsilon}_{c d}^{+}} .
\end{aligned}
$$

The last two terms in Eq. (18b) contain the product of an average and a fluctuator. By definition the average of these quantities is zero. Kröner ${ }^{21}$ has produced a solution to this problem for a disordered composite. Kröner's solution requires equilibrium at the local scale so inherently corrects the errors introduced by the mean strain approach. The approach arrives at a series solution [Eq. (19)] of increasing correlations of the local fluctuations of the stiffness. It should be noted that products of fourth-ordered tensor are of the double scalar kind: $\mathbf{A B}=A_{a b c d} B_{c d e f}$. These correlations are weighted by a mean tensor $\mathbf{R}$ whose nonzero components shown in Eq. (20) are factors of the mean Lamé constants $\bar{\lambda}$ and $\bar{\mu}$. This of course requires that the material is macroscopically isotropic

$$
\begin{aligned}
\overline{\mathbf{Z}^{+} \boldsymbol{\epsilon}^{+}}= & -\overline{\mathbf{Z}^{+} \mathbf{R} \mathbf{Z}^{+}}+\overline{\mathbf{Z}^{+} \mathbf{R Z} \mathbf{R}^{+} \mathbf{R} \mathbf{Z}^{+}} \\
& -\overline{\mathbf{Z}^{+} \mathbf{R} \mathbf{Z}^{+} \mathbf{R} \mathbf{Z}^{+} \mathbf{R Z}}+\ldots, \\
R_{1111}= & R_{2222}=\frac{\bar{\lambda}+5 \bar{\mu}}{8 \bar{\mu}(\bar{\lambda}+2 \bar{\mu})}, \\
R_{1122}= & R_{2211}=\frac{-(\bar{\lambda}+\bar{\mu})}{8 \bar{\mu}(\bar{\lambda}+2 \bar{\mu})}, \\
R_{1212}= & R_{1221}=R_{2112}=R_{2121}=\frac{\bar{\lambda}+3 \bar{\mu}}{8 \bar{\mu}(\bar{\lambda}+2 \bar{\mu})} .
\end{aligned}
$$

The series solution is good for small fluctuations and approaches a limit for a perfectly disordered material. Perfect disorder implies that the domain over which the local stiffness is defined is infinitely small when compared to the extent of the macroscale. In practice only the first term is required for a good correction to the mean strain value. In any case higher terms are difficult to calculate since more information is required about the statistical makeup of the material in question. In two dimensions the isotropic elastic stiffness has the following nonzero elements:

$$
\begin{aligned}
& Z_{1111}=Z_{2222}=\lambda+2 \mu, \\
& Z_{1122}=Z_{2211}=\lambda, \\
& Z_{1212}=Z_{1221}=Z_{2112}=Z_{2121}=\mu .
\end{aligned}
$$

Assuming that this holds at the short lengthscale as well as the macroscale the correlation $\mathbf{Z}^{+} \mathbf{Z}^{+}$in the first term of Eq. (19) will contain the terms of the fluctuations of the Lamé constants: $\overline{\left(\lambda^{+}\right)^{2}}, \overline{\left(\mu^{+}\right)^{2}}$ and $\overline{\lambda^{+} \mu^{+}}$. It must be emphasized that these results apply to any material that has local fluctuations and the ability to define a continuous stiffness at a length scale that is significantly smaller than the macroscale. This technique has been successfully implemented by Gaspar and Koenders ${ }^{22}$ where a significant advance is made in the prediction of the shear modulus of granular assemblies. To find the values of $\overline{\mathbf{Z}}$ and of the correlations $\overline{\mathbf{Z}^{+} \mathbf{Z}^{+}}$, data are 


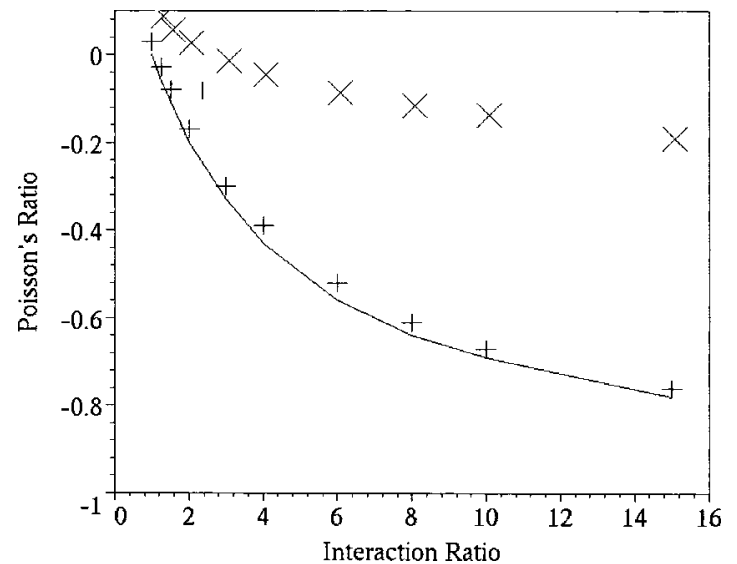

FIG. 1. Poisson ratio as a function of microscale interaction ratio. Bathurst and Rothenburg's model (solid); mean strain calculation $(+)$; heterogeneous calculation $(\times)$.

taken from a 2D simulation of a densely packed granular assembly by Kuhn. ${ }^{23-25}$ The particle positions and interactions were taken from Kuhn's simulation and used to calculate the local continuum stiffness via a novel technique. ${ }^{26,27}$ This technique fits polynomials to the spin and displacement of a small assembly of particles and the fitting criteria are based on the equilibrium equations. By associating the first displacement gradient with a strain, and calculating a stress from a volume density of forces, a relationship is obtained between the stress and strain of a small assembly of the material. From the field of $\mathbf{Z}(\mathbf{x})$ the correlations $\overline{\mathbf{Z}^{+} \mathbf{Z}^{+}}$can be found and Kröner's homogenization technique applied.

The process is carried out here, but the simulation data are treated as an auxetic material by altering the interaction between grains to induce auxetic behavior predicted by Bathurst and Rothenburg. ${ }^{11}$ A mean-strain modulus is calculated that can be compared to the Bathurst and Rothenburg's prediction in Eq. (6). A heterogeneous modulus is also calculated by adding the first term of Kröner's expansion [Eq. (19)] to the mean-strain value. These results are shown in Figs. 1, 2, and 3.

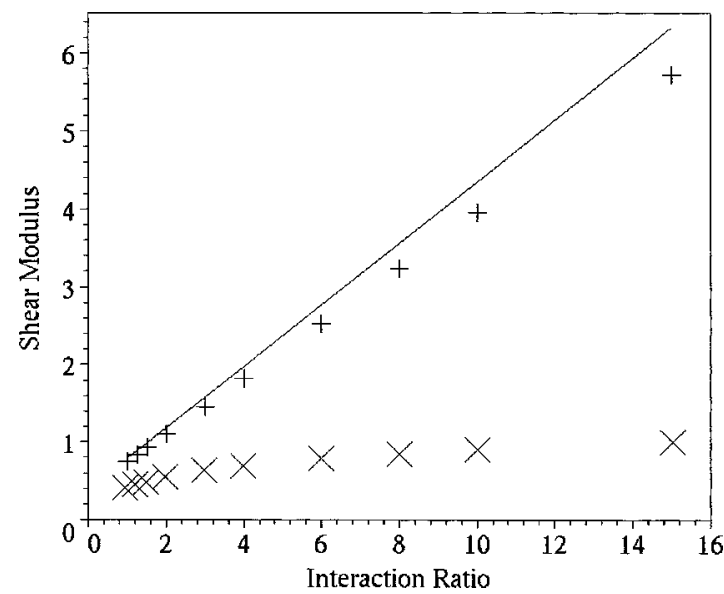

FIG. 2. Shear modulus as a function of microscale interaction ratio. Bathurst and Rothenburg's model (solid); mean strain calculation $(+)$; heterogeneous calculation $(\times)$.

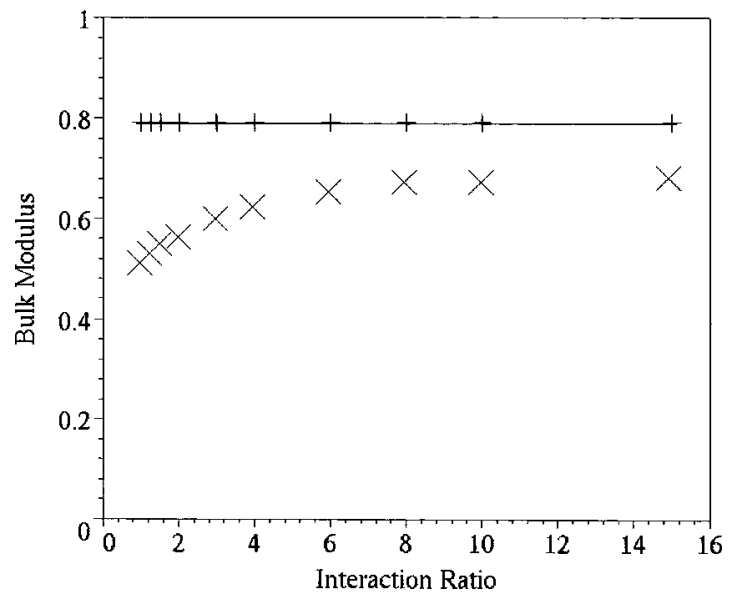

FIG. 3. Bulk modulus as a function of microscale interaction ratio. Bathurst and Rothenburg's model (solid); mean strain calculation $(+)$; heterogeneous calculation $(\times)$.

In all three figures the mean-strain calculation agrees well with Bathurst and Rothenburg which is unsurprising as Koenders and Jenkins technique is very similar to that of Bathurst and Rothenburg. However, the heterogeneous values are significantly different. According to Eqs. (5) and (6) the mean-strain Poisson's ratio as a function of interaction ratio $\xi$, will approach an asymptote of $\overline{\boldsymbol{\nu}}(\infty) \rightarrow-1$. The heterogeneous calculation similarly has an asymptote but is much reduced at $\boldsymbol{\nu}(\infty) \rightarrow-0.2$. This shows that as with conventional granular media, considerations of the heterogeneity of the material are important to for understanding the elastic response of a material with localized disorder. The shear modulus although predicted to increase linearly with $\xi$ by mean strain assumptions, increases initially but ceases to vary much with $\xi$ for large values of $\xi$. An important implication is that the large-scale elastic moduli are constrained by their fluctuations through the material. For example, in this case, the shear modulus cannot be raised above a limit by increasing $\xi$ indefinitely. The limit is determined by the fluctuations of elastic moduli. The bulk modulus whose mean strain prediction is a constant, where fluctuations do occur within the material, becomes a function of the microstructure.

\section{ANALYTICAL APPROACH IN 2D}

A two-dimensional specialization of Kröners method to calculate $\overline{\mathbf{Z}^{+} \boldsymbol{\epsilon}^{+}}$shown in Gaspar $^{27}$ produces slightly more transparent results. Equations (22)-(24) show the calculation of the principal moduli with the mean-strain and heterogeneous correction clearly separated

$$
\begin{aligned}
Z_{1111}=Z_{2222}= & \bar{\lambda}+2 \bar{\mu}-\frac{\overline{\left(\lambda^{+}\right)^{2}}+2 \overline{\left(\lambda^{+} \mu^{+}\right)}}{\bar{\lambda}+2 \bar{\mu}} \\
& -\frac{\overline{\left(\mu^{+}\right)^{2}}(\bar{\lambda}+5 \bar{\mu})}{2 \bar{\mu}(\bar{\lambda}+2 \bar{\mu})},
\end{aligned}
$$




$$
\begin{aligned}
Z_{1122}=Z_{2211}= & \bar{\lambda}-\frac{\overline{\left(\lambda^{+}\right)^{2}}+2 \overline{\left(\lambda^{+} \mu^{+}\right)}}{\bar{\lambda}+2 \bar{\mu}} \\
& +\frac{\overline{\left(\mu^{+}\right)^{2}}(\bar{\lambda}+5 \bar{\mu})}{2 \bar{\mu}(\bar{\lambda}+2 \bar{\mu})}, \\
Z_{1212}=Z_{2121}= & Z_{1221}=Z_{2112}=\bar{\mu}-\frac{\overline{\left(\mu^{+}\right)^{2}}(\bar{\lambda}+5 \bar{\mu})}{2 \bar{\mu}(\bar{\lambda}+2 \bar{\mu})} .
\end{aligned}
$$

The fluctuations of the material are captured in the correlations of the Lamé constants: $\overline{\left(\lambda^{+}\right)^{2}}, \overline{\left(\mu^{+}\right)^{2}}$ and $\overline{\lambda^{+} \mu^{+}}$. Information on these quantities is then required to understand the effect of disorder within the material. These can be normalized by $\bar{\mu}$ and $\overline{\left(\mu^{+}\right)^{2}}$.

$$
\begin{aligned}
& Z_{1111}=Z_{2222}=\bar{\mu}\left[\bar{\chi}+2+k\left(-\frac{A+2 B}{\bar{\chi}+2}-\frac{\bar{\chi}+5}{2(\bar{\chi}+2)}\right)\right], \\
& Z_{1122}=Z_{2211}=\bar{\mu}\left[\bar{\chi}+k\left(-\frac{A+2 B}{\bar{\chi}+2}+\frac{\bar{\chi}+5}{2(\bar{\chi}+2)}\right)\right], \\
& Z_{1212}=Z_{2121}=Z_{1221}=Z_{2112}=\bar{\mu}\left[1-k\left(\frac{\bar{\chi}+3}{2(\bar{\chi}+2)}\right)\right],
\end{aligned}
$$

where

$$
A=\frac{\overline{\left(\lambda^{+}\right)^{2}}}{\overline{\left(\mu^{+}\right)^{2}}}, \quad B=\frac{\overline{\lambda^{+} \mu^{+}}}{\overline{\left(\mu^{+}\right)^{2}}}, \quad k=\frac{\overline{\left(\mu^{+}\right)^{2}}}{\overline{\mu^{2}}}, \quad \chi=\frac{\bar{\lambda}}{\bar{\mu}} .
$$

A typical auxetic foam sample has a $\bar{\nu}=-0.5$. From isotropic elasticity this gives $\chi=-2 / 3$. Values for $A$ and $B$ are difficult to arrive at. These are dependent on the microstructure of the material and have the potential to vary greatly. However in 2D random networks like granular materials and honeycombs, the constraint of topological stability would require a fluctuation in one elastic modulus to influence the other. This would imply a correlation between $\lambda$ and $\mu$ and little change in $A$ and $B$ from one material of this type to the next. The values can be calculated by any technique that gives the position dependent elastic modulus for a material sample. Using the granular simulation mentioned in Sec. V with the technique by Koenders and Jenkins, ${ }^{26,27}$ values of $A$ and $B$ are calculated to be: $A=0.6$ and $B=-0.3$. In support of treating these as constants it should be noted that an isotropic elastic material that has $\nu \longrightarrow-1$ will have $\lambda / \mu \rightarrow-1$. So assuming that the fluctuations of a variable are roughly proportional to the mean, for a region of fixed Poisson's ratio, we can say

$$
\overline{\left(\lambda^{+}\right)^{2}} \sim \overline{\left(\mu^{+}\right)^{2}} \propto-\left(\overline{\lambda^{+} \mu^{+}}\right) .
$$

The similarity between the two autocorrelates in Eq. (29) indicates a value of $A$ to be about unity, which supports the calculated value of $A=0.6$. The constant of proportionality in Eq. (29) is bound between zero if $\lambda$ and $\mu$ are completely independent and one if $\lambda$ and $\mu$ are perfectly correlated and of equal mean. The reality, as already has been argued, will be a modest correlation and so a calculated value of $B$ $=-0.3$ is quite reasonable. Considering these arguments, the calculated values of $A$ and $B$ can be considered representative of this class of materials.

Taking this further it can be seen that terms containing $A+2 B$ will then become small and the corrections will be mainly dependent on the fluctuations in shear modulus. Since the auxetic response can be considered a feature of a material with a relatively large shear modulus it is not surprising that fluctuations in the shear modulus will have a dominant effect on the grand assembly moduli. If this is the case that terms in $A+2 B$ become small for a general auxetic material, then this approach is well founded for general auxetic materials.

Using these assumptions the variance of the shear modulus, $\overline{\left(\mu^{+}\right)^{2}}$, is then a measure of the fluctuations within the material. Some simple analysis can then take place on the variation of the heterogeneous moduli with changes in the level of disorder as quantified by $k$. Using these values the relative correction to the elastic moduli can be calculated as a function of the level of disorder $k$ in the material. All the isotropic moduli can be obtained from Eqs. (25)-(27) via the standard relations.

The relative corrections to the elastic moduli as obtained from Eqs. (22)-(24) are

Poisson's ratio:

$$
\frac{\nu^{\mathrm{corr}}}{\bar{\nu}}=\frac{-2\left(-2 A-4 B+\chi^{2}+6 \chi+5\right) k}{\chi\left(-2 \chi^{2}-8 \chi-8+2 k A+4 k B+k \chi+5 k\right)},
$$

Lamé's:

$$
\frac{\lambda^{\mathrm{corr}}}{\bar{\lambda}}=\frac{k(-2 A-4 B+\chi+5)}{2 \chi(\chi+2)}
$$

shear modulus:

$$
\frac{\mu^{\mathrm{corr}}}{\bar{\mu}}=-\frac{k(\chi+3)}{2(\chi+2)}
$$

Young's modulus:

$$
\frac{E^{\mathrm{corr}}}{\bar{E}}=\frac{-\left(-3 k A-6 k B-3 k+\chi^{3}+5 \chi^{2}-k \chi-k A \chi-2 k B \chi+2 A+4 B+7 \chi+5\right) k}{(\chi+1)\left(2 \chi^{2}+8 \chi+8-2 k A-4 k B-k \chi-5 k\right)},
$$



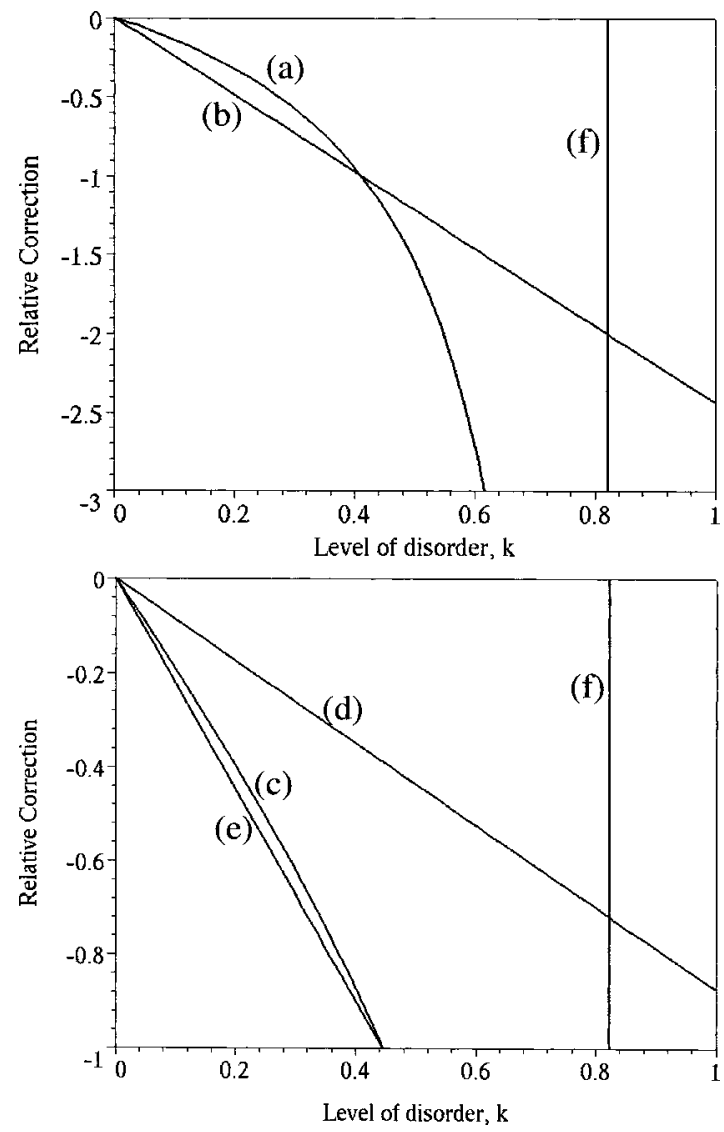

FIG. 4. Relative correction of elastic moduli as a function of disorder: Poisson's ratio (a); Lamé's constant, $\lambda$ (b); Young's modulus, (c); shear modulus (d); bulk modulus (e); and asymptotes for curved graphs (f).

bulk modulus:

$$
\frac{K^{\mathrm{corr}}}{\bar{K}}=-\frac{k(A+2 B+1)}{(\chi+1)(\chi+2)} \text {. }
$$

For constant $A, B$, and $\chi$ these relative corrections are plotted in Figs. 4(a)-4(e). It is worth noting that the measure of disorder $k$ is the variance of shear modulus and is a square measure of the disorder. The corrections shown in Eqs. (30)(34) are proportional to $k$ and so like Fortes and Ashby's results are proportional to a square measure of disorder. Figures 4(a) and 4(b) show the Lamé constant $\lambda$ and Poisson's ratio. An asymptote at $k=0.8$ is shown by a vertical in the same lines style as the function to which it pertains. Since these moduli can be positive or negative, the relative correction can be larger than -1 . A material manufactured to be auxetic, therefore, will become conventional when the relative correction to the Poisson's ratio reaches -1 or $\nu^{\text {corr }}$ $=-\bar{\nu}$. The solution to Eq. (30) for $\nu^{\text {corr }}=-\bar{\nu}$ gives the level of fluctuations that turns an auxetic material conventional and is shown in Eq. (35)

$$
k=\frac{2 \chi(\chi+2)}{2 A+4 B-\chi-5} .
$$

For the values of $\chi, \mu, A$, and $B$ used above this returns $k$ $\approx 0.41$. For small fluctuations, i.e., $k<0.2$, the relative cor- rection the the Poisson's ratio is roughly linear with zero intercept and a gradient of -0.25 . So a suitable approximation is

$$
\nu=\bar{\nu}(1-1.65 k) \text {. }
$$

The equivalent for the Lamé constant $\lambda$ is

$$
\lambda=\bar{\lambda}(1-2.50 k) \text {. }
$$

The other principal elastic moduli, Young's, shear, and bulk, are required to always be positive. Hence when the relative correction reaches -1 this approach predicts that the modulus of elasticity will become undefined. A physical interpretation is that the idea of static equilibrium cannot be attained and the material enters a regieme dependent on the material microstructure. For example, a foam may develop regions of collapsed cells, whereas a granular material may exhibit dynamic failure such as slip deformation. ${ }^{28}$ It is questionable how valid this model is for large values of $k$ since the series expansion in Eq. (19) generally works best for small $k$. If it is accepted for large $k$ then the model predicts an undefined elastic modulus when the first relative correction reaches -1 . From Fig. 4(c) this is the Bulk and the Young's modulus and the exact point can be calculated from

$$
k=\frac{(\chi+2)(\chi+1)}{A+2 B+1},
$$

which for the values of $\chi, \mu, A$, and $B$ used above evaluates to $k \approx 0.44$ Another solution is possible for the Young's modulus in the region $k>1$ but has been discarded since it is way beyond the validity of the homogenization process. For these moduli the small fluctuation approximations are:

$$
\begin{aligned}
E & =\bar{E}(1-2.0 k), \\
\mu & =\bar{\mu}(1-0.88 k), \\
K & =\bar{K}(1-2.25 k) .
\end{aligned}
$$

Should the model only be considered over small $k$ then the gradients of the linear approximations [Eqs. (36)-(37) and (39)-(41)] give a useful insight. Where a material is being manufactured for a specific elastic modulus, the fluctuations within the material give an element of uncertainty as to the exact modulus of the final material. The linear approximations to the heterogeneous model indicate that the moduli that are most sensitive are Lamé's constant $\lambda$, bulk modulus, and Young's modulus. Conversely the shear modulus and Poisson's ratio are the most stable.

\section{DISCUSSION AND CONCLUSIONS}

It is attractive to use mean-field models when investigating complex materials since perfectly ordered materials are rarely seen or used. However, even if a mean-field model does produce results comparable to experiment, it is unclear whether the parameters in the model reflect the microstructure of the material. In granular materials, mean field models have been found fundamentally inadequate. ${ }^{27}$ In the emerging topic of auxetic materials, mean-field models are widely used to represent fundamentally disordered materials such as foams. Calculations that extend from granular mechanics in- 
dicate that the fluctuations within the material impose a large correction to the mean-field response of the Poisson's ratio among other moduli. An elementary 2D calculation of the magnitude of the correction to the mean-field response due to the level of disorder shows that it takes a little over 30\% fluctuations in the shear modulus to reduce the magnitude of the Poisson's ratio by $50 \%$ for an initial Poisson's ratio of $\nu \approx-0.5$. This information has important ramifications on the design of auxetic materials. Conversely, for an isotropic foam with a Poisson's ratio of $\nu \approx-0.7$, the correction to the mean strain must be quite small, $30 \%$ at a theoretical maximum. This would indicate that there is less than a $10 \%$ fluctuation in the elastic moduli of the material. This may seem quite small when considering the disorder that can be seen visually in something like a foam sample. This work is focused on a 2D isotropic material and a 2D specialization of Kröner's homogenization technique. The extension to 3D would remove the transparency but could nevertheless return useful information. Torquato ${ }^{29}$ uses the approach of spherical inclusions in a matrix to calculate the moduli of an effective medium. This approach works best for dillute concentrations of inclusions where no interaction is considered between inclusions. The first term of Kröner's expansion in Eq. (19) contains the two-point correlation function of the stiffness fluctuations so includes interactions between two inclusions or stiffness points. This is an advantage for modeling a material that has a modulus that varies continuously.

Figure 4 shows that the bulk scale moduli when corrected for disorder have differing responses to the level of disorder. This is important to consider when characterizing a material. In this case the Poisson's ratio and Lamé constant $\lambda$ are more sensitive to fluctuations in a material than the Young's, bulk, and shear moduli. For example, when a set of samples of a material are being charceterized, the Poisson's ratio is a more sensitive measure of consistancy between samples than the Young's modulus.

One other question remains unanswered: Is it valid to define an elastic moduli over a single foam cell? The elastic moduli are a relationship between the stress and strain or displacement gradient. If this gradient cannot be defined then the strain and therefore elastic moduli cannot be defined. In a material that contains sharp steps between materials such as the ribs in a foam or voids in a granular material, it is not clear whether the displacement gradient has a definition. If a large enough sample of the material is used then the steps between materials become insignificant and these elastic variables can be used. What is then required is a means to find out how large this sample of the material has to be. Koenders ${ }^{30}$ provides a key to answering this question by predicting spatial features within a heterogeneous material under a persistent strain path. These spatial features lead to a length measurement of the continuum scale within the mate- rial. When applied to granular mechanics, the continuum length was found to be about 5 mean grain diameters. ${ }^{27}$ This explains why theoretical work in granular mechanics that only considers a distribution of single contacts has been unsuccessful. ${ }^{3,27}$ Theoretical work that is based on a continuum scale of 5 grain diameters is successful and is able to reasonably predict the elastic moduli of granular material. ${ }^{22}$ Since this continuum length lies between the microscale of the material and the macroscale, it is useful to term it the mesoscale. As yet there has not been any work to find the continuum length of any of the typical auxetic materials or even to characterize dominant length scales in classical foams. Further work by the authors is underway to determine this continuum length scale within auxetic foams.

\section{ACKNOWLEDGMENT}

Financial support from the EPSRC, Grant No. GR/ R84870/01 is gratefully acknowledged.

${ }^{1}$ A. E. H. Love, A Treatise on the Mathematical Theory of Elasticity (Dover, 1927).

${ }^{2}$ K. E. Evans and A. Alderson, Adv. Mater. (Weinheim, Germany) 12, 617 (2000).

${ }^{3}$ N. Gaspar and M. A. Koenders, J. Eng. Mech. 127, 10, 987 (2001).

${ }^{4}$ R. H. Baughman, J. M. Shacklette, A. A. Zakhidov, and S. Stafstrom, Nature (London) 392, 362 (1998).

${ }^{5}$ B. M. Lempriere, AIAA J. 6, 2226 (1968).

${ }^{6}$ N. Gaspar and M. A. Koenders (private communication, 2002).

${ }^{7}$ B. D. Caddock and K. E. Evans, J. Phys. D 22, 1877 (1989).

${ }^{8}$ R. Lakes, Science 235, 1038 (1987).

${ }^{9}$ K. L. Alderson and K. E. Evans, Polymer 33, 4435 (1992).

${ }^{10}$ A. P. Pickles, K. L. Alderson, and K. E. Evans, Polym. Eng. Sci. 36, 636 (1996).

${ }^{11}$ R. J. Bathurst and L. Rothenburg, Int. J. Eng. Sci. 26, 4 (1988).

${ }^{12}$ D. H. Boal, U. Seifert, and J. C. Shillcock, Phys. Rev. E 48, 4274 (1993).

${ }^{13}$ D. R. Veronda and R. A. Westmann, J. Biomech. 3, 111 (1970).

${ }^{14}$ N. Chan and K. E. Evans, J. Cell. Plast. 34, 231 (1998).

${ }^{15}$ M. Burke, New Sci. 154, 36 (1997).

${ }^{16}$ D. Weaire and N. Rivier, Contemp. Phys. 25, 1 (1984).

${ }^{17}$ I. G. Masters and K. E. Evans, Compos. Struct. 35, 403 (1996).

${ }^{18}$ C. W. Smith, J. N. Grima, and K. E. Evans, Acta Mater. 48, 4349 (2000).

${ }^{19}$ L. Rothenburg, A. A. Berlin, and R. J. Bathurst, Nature (London) 354, 470 (1991).

${ }^{20}$ M. A. Fortes and M. Ashby, Acta Mater. 47, 3469 (1999).

${ }^{21}$ E. Kroner, J. Mech. Phys. Solids 15, 319 (1967).

${ }^{22}$ N. Gaspar and M. A. Koenders, Proceedings of the 4th International Conference on Micromechanics of Granular Media: Powders and Grains, 2001, p. 165

${ }^{23}$ M. R. Kuhn, in Mechanics Computing in 1990's and Beyond, edited by H. Adeli and R. L. Sierakowski (1991), pp. 1229-1233.

${ }^{24}$ M. R. Kuhn, Mech. Mater. 31, 407 (1999).

${ }^{25}$ M. R. Kuhn (private communication, 2000).

${ }^{26}$ M. A. Koenders and J. T. Jenkins (private communication).

${ }^{27}$ N. Gaspar, PhD thesis, Kingston University, 2002.

${ }^{28}$ J. R. F. Arthur and T. Dunstan, IUTAM Conference on Deformation and Failure of Granular Materials, 1982, pp. 453-459.

${ }^{29}$ S. Torquato, Random Heterogeneous Materials (Springer, New York, 2001).

${ }^{30}$ M. A. Koenders, Phys. Rev. E 56, 5585 (1997). 